\title{
Magnetic Resonance Imaging Analysis of Moving Fronts in Floating Dosage Forms
}

\author{
P. Kulinowski ${ }^{a, *}$, P. DorożyŃski ${ }^{b}$, R. JACHOWiCZ ${ }^{b}$ \\ AND A. JASIŃSKI ${ }^{a, c}$ \\ ${ }^{a}$ Department of Magnetic Resonance, Institute of Nuclear Physics PAS \\ Radzikowskiego 152, 31-342 Kraków, Poland \\ ${ }^{b}$ Department of Pharmaceutical Technology and Biopharmaceutics \\ Pharmaceutical Faculty, Jagiellonian University Medical College \\ Medyczna 9, 30-688 Kraków, Poland \\ ${ }^{c}$ Pedagogical University, Podchorạżych 2, 30-084 Kraków, Poland
}

An application of magnetic resonance imaging in the field of pharmaceutical technology is presented in this paper. The analysis of diffusion and swelling fronts was carried out for four floating dosage forms using magnetic resonance imaging. The influence of polymer viscosity, its concentration, and type of applied dissolution media on the area of moving fronts was investigated.

PACS numbers: $87.61 .-\mathrm{C}$

\section{Introduction}

Floating systems could be useful for controlled delivery of drugs which act locally in the stomach or have a narrow absorption window in the proximal part of the gastrointestinal tract. Among the floating systems hydrodynamically balanced systems (HBS) are the only commercially available formulations. HBS are essentially composed of a drug intimately mixed with gel forming hydrocolloids, which swell in contact with gastric fluid. The hydrogel shell maintains a relative integrity of shape and has a bulk density smaller than $1 \mathrm{~g} / \mathrm{cm}^{3}$. The drug is slowly released in the stomach by diffusion through the hydrogel barrier and erosion of the surface of the dosage form.

*corresponding author 
In the hydrophilic matrices, drug release is determined by swelling and erosion of the polymer matrix and dissolution of the drug. The kinetics of these phenomena can be analysed by the observing of the motion of swelling and diffusion fronts within the matrix. The positions of the diffusion and swelling fronts define the hydrogel layer thickness. This is the fundamental factor which controls drug release from the dosage form. The position of the swelling front defines the border of the inner dry core which controls the flotation of the HBS formulation.

The aim of this work was to determine how viscosity and concentration of the polymer and the applied dissolution medium affect the position of the fronts in the gel layer of HBS.

\section{Materials}

Hypromellose (HPMC) is a partly O-methylated and O-(2-hydroxypropylated) cellulose widely used in pharmaceutical technology. In this study HPMC with two viscosities $400 \mathrm{cP}$ and $100000 \mathrm{cP}$ were used. The model drug employed was L-dopa. White opaque hard gelatin capsules Coni-Snap ${ }^{\circledR}$ size 1 and 0 were used. All reagents were in at least p.p.a. grade. Mixtures of L-dopa and HPMC in the ratios 1:1 and 1:3 were prepared in the mortar. Capsules were filled manually with non-compressed powder mixtures corresponding to $100 \mathrm{mg}$ of L-dopa.

For swelling studies two kinds of media were used: fasted state simulated gastric fluid (FaSSGF) - hydrochloric acid $0.1 \mathrm{M}$ and fed state simulated gastric fluid (FeSSGF) - hydrochloric acid $0.01 \mathrm{M}$ with addition of sodium laurylsulphate $(2.5 \mathrm{~g} / \mathrm{l})$ and sodium chloride $(2.0 \mathrm{~g} / \mathrm{l})$.

In this study the following cases were analyzed:

- metolose $100000 \mathrm{cP}+$ L-dopa (3+1) in FaSSGF,

- metolose $100000 \mathrm{cP}+$ L-dopa (3+1) in FeSSGF,

- metolose $100000 \mathrm{cP}+$ L-dopa (1+1) in FeSSGF,

- metolose $400 \mathrm{cP}+$ L-dopa $(3+1)$ in FeSSGF.

\section{Methods}

The magnetic resonance imaging (MRI) studies were done with a research MRI system consisting of a digital console MARAN DRX and a $4.7 \mathrm{~T} / 310 \mathrm{~mm}$ horizontal bore superconducting magnet equipped with an actively shielded gradient set of $200 \mathrm{~mm}$ ID [1]. A specially designed, MR compatible, flow-through cell combined with MRI probe-head for HBS systems investigation was used [2]. In the cell the HBS capsule was kept in a horizontal position in the centre of the $\mathrm{RF}$ coil, and was oriented along the direction of $B_{0}$ field. The experiments were carried out under continuous flow in two dissolution media, FaSSGF and FeSSGF. 
MR images were obtained every half an hour with a flow compensated spin-echo (SE) pulse sequence to avoid effects of flowing solvent in MR images. Back-to-back bipolar gradient wave forms (shaded areas in Fig. 1) were used in velocity insensitive SE sequence for gradient first moment nulling [3]. Single experiment lasted for 5 hours.

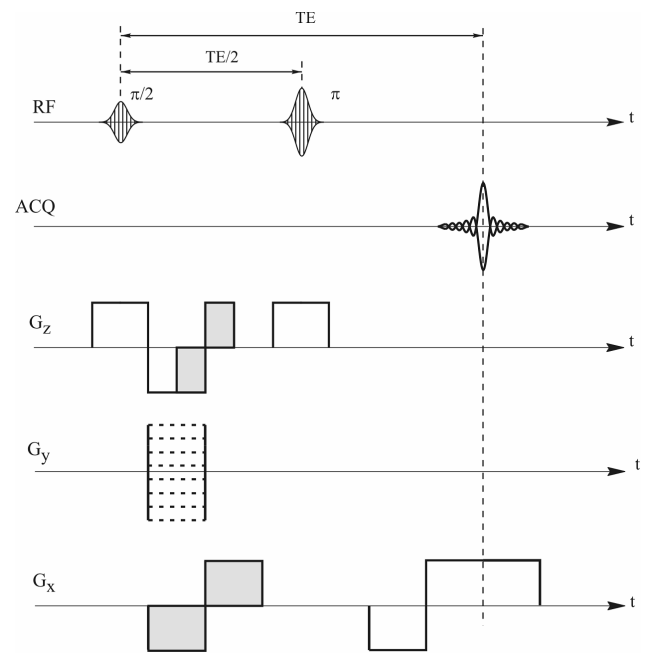

Fig. 1. Flow compensated spin-echo MRI pulse sequence $(\mathrm{RF}-\pi$ and $\pi / 2$ radio frequency pulses; ACQ - acquired MR signal; $G_{x}, G_{y}, G_{z}$ - magnetic field gradient pulses in $x, y, z$ directions, respectively; TE - echo time).

Areas of different HBS sections, i.e. hydrogel, dry core were measured in MR images with the "ImageJ" software (NIH, Bethesda, MD, USA).

\section{Results}

The formation of moving fronts was imaged in this study by MRI. Figure 2 shows examples of the identification moving front for HPMC $100000 \mathrm{cP}$ and L-dopa mixed in a 3:1 ratio in FeSSGF after 5 hours.

A comparative analysis of the images shows that the mechanism of water penetration into the polymer matrix is related to viscosity of the polymer and its concentration in the dosage form. The MR images of HBS show not only the general shape of the capsule but also its structural characteristics.

In the case of HBS containing HPMC $100000 \mathrm{cP}$ and L-dopa in 3:1 ratio in FaSSGF, a rapid increase in the area of the diffusion front was observed in the images upon placement in the solvent (see Fig. 3). The area of the diffusion front increased from 500 pixels after 60 minutes to almost 2000 pixels after 90 minutes. For the following 4 hours the area of the diffusion front remained nearly constant. In the other cases the area of diffusion fronts increased gradually during five hours. 

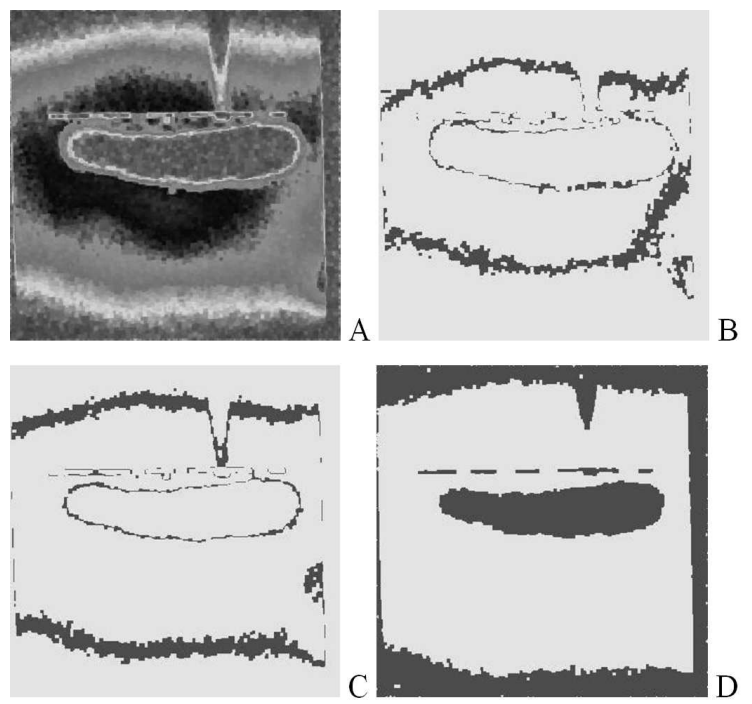

Fig. 2. Examples of the identification of moving fronts: (A) original image, (B) diffusion front, (C) swelling front, (D) dry core.

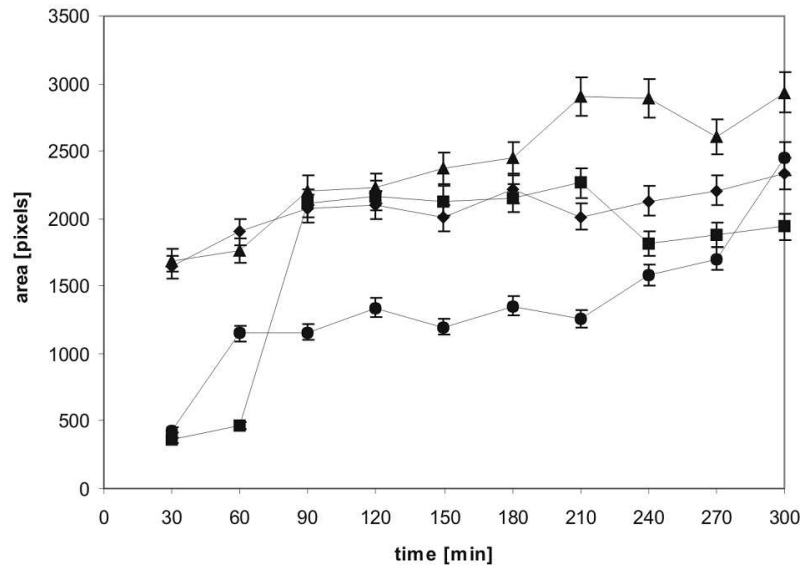

Fig. 3. Profiles of changes of areas of diffusion fronts: $\boldsymbol{\Delta}$ metolose $400 \mathrm{cP}+$ L-dopa (3+1) in FeSSGF, • metolose 100000 cP + L-dopa (1+1) in FeSSGF, metolose $100000 \mathrm{cP}+$ L-dopa $(3+1)$ in FaSSGF, metolose $100000 \mathrm{cP}+$ L-dopa $(3+1)$ in FeSSGF.

The areas of the swelling fronts increased gradually in all cases (Fig. 4). The fastest formation of the swelling front in the early stages of hydrogel formation was observed for HBS containing the mixture of HPMC $400 \mathrm{cP}$ and L-dopa in a 3:1 ratio in FeSSGF. After 30 minutes the area of the swelling front was 838 pixels and after 300 minutes it has grown to about 1300 pixels. In the case of a HBS containing mixture of HPMC $100000 \mathrm{cP}$ and L-dopa in a 3:1 ratio in FaSSGF, the 


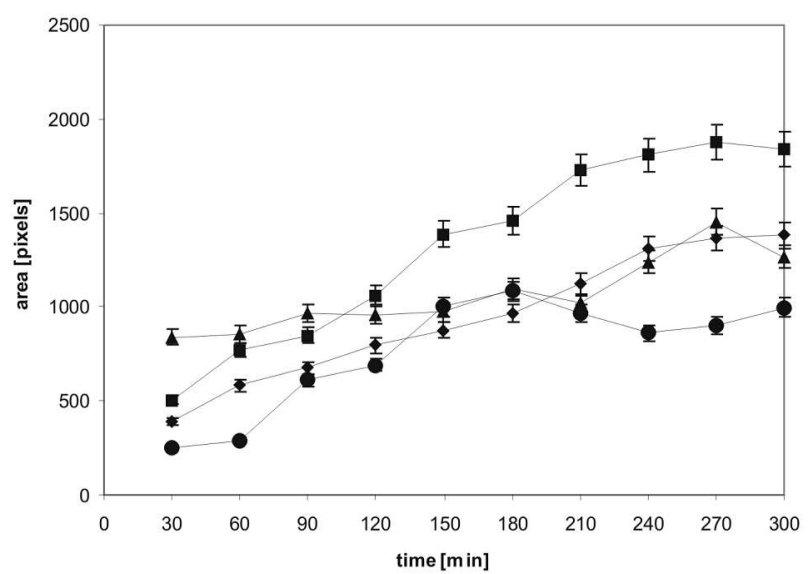

Fig. 4. Profiles of changes of areas of swelling fronts: $\boldsymbol{\Delta}$ metolose $400 \mathrm{cP}+$ L-dopa $(3+1)$ in FeSSGF, - metolose $100000 \mathrm{cP}+$ L-dopa $(1+1)$ in FeSSGF, - metolose $100000 \mathrm{cP}+$ L-dopa $(3+1)$ in FaSSGF, $\bullet$ metolose $100000 \mathrm{cP}+$ L-dopa $(3+1)$ in FeSSGF.

increase in the swelling front in the end of experiment was most effective. The area of swelling front increased by a factor of 3.5 during five hours. These observations suggest that the dissolution of the active substance and the floating properties of the HBS formulations depend on the type of the dissolution medium (FeSSGF or FaSSGF) and the concentration of the polymer in the dosage form.

An analysis of the dry cores of the HBS have shown that their areas were almost constant (Fig. 5)

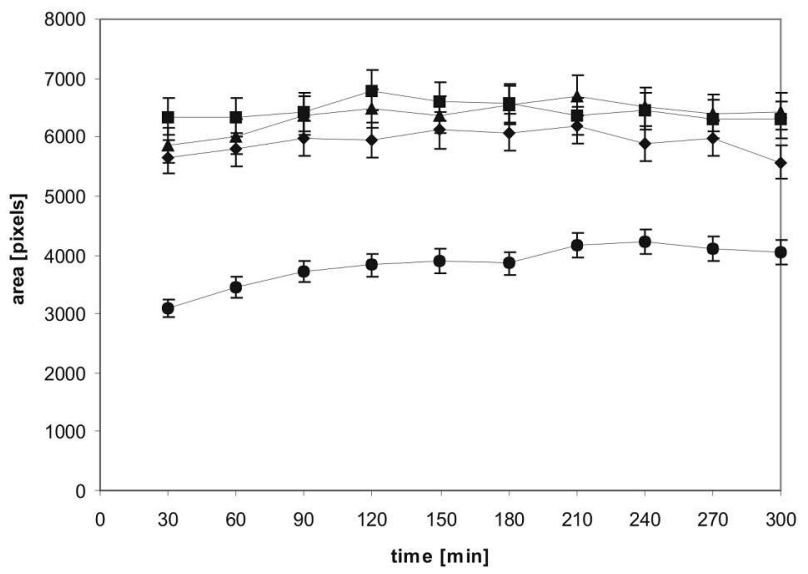

Fig. 5. Profiles of changes of areas of dry cores: $\boldsymbol{\Delta}$ metolose $400 \mathrm{cP}+\mathrm{L}$-dopa $(3+1)$ in FeSSGF, • metolose $100000 \mathrm{cP}+\mathrm{L}$-dopa $(1+1)$ in FeSSGF, metolose $100000 \mathrm{cP}+$ L-dopa $(3+1)$ in FaSSGF, $\bullet$ metolose 100000 cP + L-dopa $(3+1)$ in FeSSGF. 
Swelling and diffusion front area profiles in the hydrogel matrix and dry core area profiles can be used to describe the integrity of the HBS as well as the swelling properties of the dosage forms.

Observations of increasing areas of the swelling and diffusion fronts and almost constant areas of dry cores between 30 and 300 min after capsule immersion suggest that the dimensional changes of the HBS displace the molecules in the matrix due to expansion of polymeric chains $[4,5]$. Moreover, the area of diffusion front is almost constant between 90 and 210 min after capsule immersion. In the same time area of swelling front increases. In other words, area between the swelling and diffusion fronts decreases. It suggests that the process of hydrogel erosion takes place. It means that during hydration of the HBS under the continuous medium flow two opposite processes take place: the swelling of the polymer matrix and the erosion of the outer layer of the hydrogel. The appropriate balance between swelling and erosion processes might effectively control the floating properties of HBS and dissolution of the active substance.

Erosion of hydrogel should be confirmed using other methods used in pharmaceutical sciences.

\section{Conclusions}

1. Moving fronts can be observed with MRI.

2. The rate of swelling of uncompressed HPMC matrices depends on the type of medium, concentration of the polymer in the dosage form and the viscosity of the polymer.

3. The dissolution of L-dopa (which is soluble in water) depends mainly on the diffusion of the dissolved drug in the polymer matrix.

4. The erosion of the matrix is an additional mechanism influencing the dissolution of the drug.

\section{Acknowledgments}

The Ministry of Science and Informatics supported this work under grant No. 2P05F 00428.

\section{References}

[1] P. Dorożyński, R. Jachowicz, P. Kulinowski, S. Kwieciński, K. Szybiński, T. Skórka, A. Jasiński, Drug Dev. Ind. Pharm. 30, 947 (2004).

[2] C.A. Fyfe, H. Grondey, A.I. Blazek-Welsh, S.K. Chopra, B.J. Fahie, J. Contr. Rel. 68, 73 (2000).

[3] D.G. Nishimura, A. Macovski, J.M. Pauly, IEEE Trans. Med. Imag. MI-5, 140 (1986).

[4] I.S. Moussa, L.H. Cartilier, J. Contr. Rel. 42, 47 (1996).

[5] N.A. Peppas, P. Bures, W. Leobandung, H. Ichikawa, Eur. J. Pharm. Biopharm. 50, 27 (2000). 